\title{
Monitoring Space Shuttle Air Quality Using the Jet Propulsion Laboratory Electronic Nose
}

\author{
Margaret Amy Ryan, Hanying Zhou, Martin G. Buehler, Kenneth S. Manatt, Victoria S. Mowrey, Shannon P. Jackson, \\ Adam K. Kisor, Abhijit V. Shevade, and Margie L. Homer
}

\begin{abstract}
A miniature electronic nose (ENose) has been designed and built at the Jet Propulsion Laboratory (JPL), Pasadena, CA, and was designed to detect, identify, and quantify ten common contaminants and relative humidity changes. The sensing array includes 32 sensing films made from polymer carbon-black composites. Event identification and quantification were done using the Levenberg-Marquart nonlinear least squares method. After successful ground training, this ENose was used in a demonstration experiment aboard STS-95 (October-November, 1998), in which the ENose was operated continuously for six days and recorded the sensors' response to the air in the mid-deck. Air samples were collected daily and analyzed independently after the flight. Changes in shuttle-cabin humidity were detected and quantified by the JPL ENose; neither the ENose nor the air samples detected any of the contaminants on the target list. The device is microgravity insensitive.
\end{abstract}

Index Terms-Air quality, carbon black, electronic nose (ENose), polymer sensor, polymer composite.

\section{INTRODUCTION}

$\mathbf{T}$ HE ABILITY to monitor the constituents of breathing air is critical to maintaining human health in a closed environment. NASA is interested in developing monitoring capability for use in closed environments in which air is recycled, such as the space shuttle, the space station, and planned extraterrestrial human habitats. For prolonged space habitation, it will be necessary to monitor the breathing air continuously without major investment of crew time. Contaminants may be present in the breathing atmosphere for many reasons: compounds not easily removed by filtering and processing will remain in the atmosphere, compounds built up in the filtering systems may eventually break through, and episodic events, such as fires or spills, will generate compounds. Air must be monitored for the presence of contaminants at levels which have the potential to be harmful to crew health. These levels are defined as spacecraft maximum allowable concentrations (SMACs) for 1-h, 24-h, 7-day, and 180-day periods, and have been deter-

Manuscript received August 14, 2001; revised August 14, 2003. This work was supported in part by the Jet Propulsion Laboratory, California Institute of Technology, Pasadena, under a contract with the National Aeronautics and Space Administration, and in part by NASA Code UL, Advanced Environmental Monitoring and Control. The associate editor coordinating the review of this paper and approving it for publication was Dr. H. Troy Nagle.

The authors are with the Jet Propulsion Laboratory, California Institute of Technology, Pasadena, CA 91109-8099 USA (e-mail: Margaret.A.Ryan@ jpl.nasa.gov; Hanying.Zhou@jpl.nasa.gov; martin.g.buehler@jpl.nasa.gov; kmanatt@jpl.nasa.gov; victoria.s.mowrey@jpl.nasa.gov; Shannon.P.Jackson@ jpl.nasa.gov; ADAM.KISOR@jpl.nasa.gov; Abhijit.Shevade@jpl.nasa.gov; Margie.L.Homer@jpl.nasa.gov).

Digital Object Identifier 10.1109/JSEN.2004.827275 mined by toxicological assessments [1], [2]. Generally, the 24-h SMACs are single or fractional parts-per-million (ppm) at atmospheric pressure.

Currently, air quality monitoring on the shuttle that is done with real-time analysis include a carbon dioxide monitor, formaldehyde badges, and a compound specific analyzer for combustion products. Air quality is also evaluated in post-flight analysis by use of samples that are either collected at a specific time and place, in a grab sample canister, or samples that are collected over an extended period of time on absorbent columns. Samples of air collected from the shuttle, either during the space flight or immediately after shuttle landing, have been analyzed and recorded for more than 100 flights to date [3]. Analysis is done in an analytical chemistry laboratory using analytical instruments such as a gas chromatograph-mass spectrometer.

The best low power, low maintenance, low volume gas sensor now available and capable of detecting and identifying a broad suite of compounds is the human nose. Many devices now commercially available for broadband monitoring as required on the shuttle are limited by power and weight restrictions, as well as by the number of compounds detectable. In addition, extensive crew time is often required to operate such monitors. For instance, a gas chromatograph-mass spectrometer (GC-MS) is capable of highly accurate analysis at very low levels of contaminants, but it requires significant crew time to operate and maintain. A volatile organic compound analyzer detects the total carbon present and does not distinguish among the individual components of mixtures or identify the compounds. The need is for low power gas monitors which can identify compounds and be operated with a minimum of crew time and which can be integrated into environmental control systems.

\section{Electronic Nose (ENose) AT the JeT PROPULSION LABORATORY (JPL)}

The goal of this electronic nose (ENose) development program was to develop a miniature sensor which may be used to monitor the breathing air in the international space station and which may be integrated into or coordinated with the environmental control system. Integration into the control systems would make it possible to solve air quality problems with minimal crew intervention. Such an instrument is envisioned for use as an incident monitor, to notify the crew of the presence of potentially dangerous substances from spills and leaks, and to provide early warning of incipient fires. An event monitor such as this one may also be used as a reliable method to make judgments on the use of breathing apparatus; if the crew has put on 
breathing apparatus while repairing a leak or cleaning a spill, it is necessary to know whether it is safe to remove the apparatus. With further development and integration into the environmental control system, such a monitor would also provide notification for automated control of sudden adverse events, such as spills, leaks, or recycling filter breakthrough that result in the release of dangerous substances, as well as the slow buildup of toxic compounds.

In order to fill the need for a device capable of monitoring air quality, the Jet Propulsion Laboratory (JPL) has developed a low power, miniature gas sensor capable of distinguishing among and identifying a suite of gas species which may be present in the recirculated breathing air of the space shuttle or space station [4]-[7]. This device is an ENose, and the sensing array is made from polymer-carbon composite sensing films [4]-[9]. Polymer-carbon composite films have been used in many sensing applications, including pressure [10], temperature [11], organics in water, and analytes in air [10]-[15]. The sensing films used in the JPL ENose are based on the initial sensing film studies done in the Lewis group at the California Institute of Technology (Caltech), Pasadena [13], [14]. These sensing films are made from commercially available insulating polymers which are loaded with carbon black as the conductive medium. Film fabrication and film response characteristics have previously been discussed in detail in [4]-[9], [13], and [14]. The device development program at the JPL included further studies of sensing films, development of electronic interfaces between sensors and data acquisition units, and development of data analysis software to identify and quantify selected contaminants singly or in a mixture of two contaminants. The resulting device, the first generation JPL ENose, was operated in an experiment on space shuttle flight STS-95 in 1998 [4]-[7]. Its mass is $1.4 \mathrm{~kg}$, including the palm-top computer used for data acquisition and storage and for control, its volume is $<2000 \mathrm{~cm}^{3}$, and it consumes $1.5 \mathrm{~W}$ on average and $3-\mathrm{W}$ peak power.

The ENose flight experiment was designed to test the ability of the ENose to monitor the air in the mid-deck of the space shuttle orbiter continuously and to test its performance in microgravity. A set of possible contaminant compounds was determined and the device was trained to detect and quantify those compounds at or below the 1-h SMAC. The ENose monitored the air continuously for six days during flight STS 95 (October $29-$ November 7, 1998). Once the device was removed from the storage locker and turned on, it operated continuously, without crew intervention, except for the introduction of alcohol wipes used to validate operation.

The experiment was controlled by collecting a daily air sample in a grab sample container (GSC). The crewmember responsible for the experiment, Commander C. L. Brown, monitored the device for operation by checking the operating lights on the side of the unit, gathered an air sample for experiment control, and provided a daily event. In order to confirm that the ENose was operating, an "event" or daily marker was provided by exposing the inlet of the unit to a medical swab saturated with 70\% 2-propanol, balance unknown. Air samples in the GSCs were analyzed in the Johnson Space Center Toxicology Laboratory after the flight.
TABLE I

POLYMERS USED FOR THE SENSOR FILMS IN THE JPL ENOSE SENSING ARRAY

\begin{tabular}{c|c|l}
\hline $\begin{array}{c}\text { Sensor } \\
\text { Position }\end{array}$ & JPL ID & \multicolumn{1}{|c}{ Polymer } \\
\hline 1,2 & C71 & Poly (2, 4, 6-tribromostyrene) \\
\hline 3,4 & A & Poly (4-vinylphenol) \\
\hline 5,6 & Q & Poly (ethylene oxide) \\
\hline 7,8 & C38 & Polyamide resin \\
\hline 9,10 & C7 & Cellulose triacetate \\
\hline 11,12 & C58 & Poly (2-hydroxyethyl methacrylate) \\
\hline 13,14 & C90 & Vinyl alcohol/ vinyl butyral copolymer, $80 \%$ vinyl butyral \\
\hline 15,16 & E15 & Poly (caprolactone) \\
\hline 17,18 & C & Poly (vinylchloride-co-vinyl acetate) \\
\hline 19,20 & D & Poly (vinyl chloride-co-vinyl acetate) $10 \%$ vinyl acetate \\
\hline 21,22 & E & Poly (vinyl acetate) \\
\hline 23,24 & F & Poly (N-vinylpyrrolidone) \\
\hline 25,26 & C88 & Styrene/isoprene, 14/86 ABA Block copolymer \\
\hline 27,28 & C80 & Poly (vinyl stearate) \\
\hline 29,30 & C22 & Methyl vinyl ether/ maleic acid 50/50 copolymer \\
\hline 31,32 & C20 & Hydroxypropyl methyl cellulose, 10/30 \\
\hline
\end{tabular}

\section{EXPERIMENTAL}

\section{A. Materials}

The polymers used to make the films are listed in Table I. Polymers C71, C38, C7, C58, C90, C88, C80, C22, and C20 were purchased from Scientific Polymer Products, Inc. Polymers A, D, E, and Q were purchased from Polysciences, Inc. Polymer $\mathrm{F}$ was purchased from Fisher Scientific and polymer C was purchased from Aldrich. The carbon black used in the films was Black Pearls 2000, a furnace black made by the Cabot Corporation. All of the solvents used to dissolve the polymers and disperse the carbon black were reagent grade solvents from $\mathrm{J}$. T. Baker and were used as received. Both the polymers and the carbon black were also used as received.

For testing the array response to analytes, the Freon was 1,1,2-trichlorotrifluoro ethane, HPLC grade, from Sigma-Aldrich, and the ethanol was from EM Science. Ammonium hydroxide was purchased from J. T. Baker, and a diluted solution of 12:1, ammonium hydroxide:water, by volume, was used to expose the films to ammonia. All other chemicals used in testing the sensor arrays were reagent grade chemicals purchased from J.T. Baker and were also used as received.

\section{B. Sensors}

The sensors in the JPL ENose are thin films made from insulating, commercially available polymers which have been loaded with carbon black as a conductive medium. A baseline resistance of each film is established; as the constituents in the air change, the films swell or contract in response to the new composition of the air, and the resistance increases or decreases. Sensing films were deposited on ceramic substrates which had eight Au-Pd electrode sets. The sensor substrate is $25 \times 10 \mathrm{~mm}$; each sensing film covered an electrode set with an area of $2 \times 1 \mathrm{~mm}$. A photo of a sensor substrate with deposited films is shown in Fig. 1.

Four sensor substrates were used in the device for a total of 32 sensors. In this experiment, 16 polymers were used to make sensing films; each polymer was used to make two sensors. The 


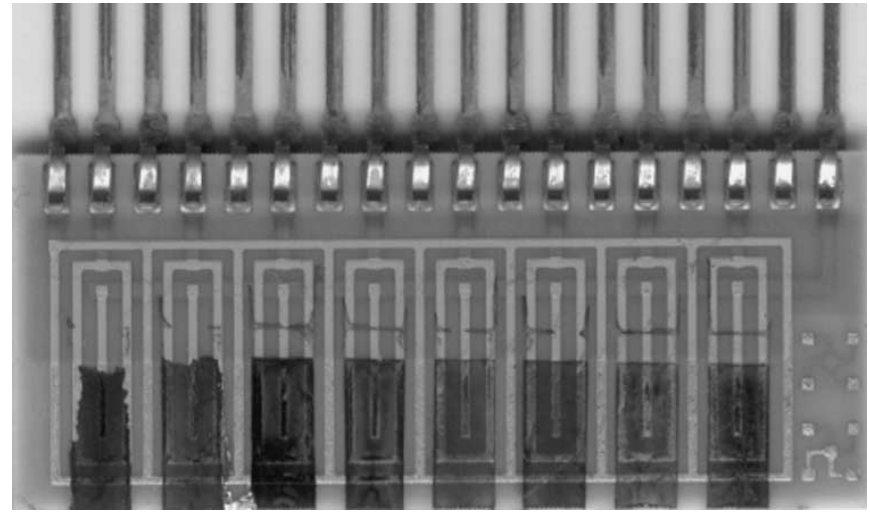

Fig. 1. Photograph of the ceramic substrate with eight sensor films.

polymers used in the ENose flight experiment were selected by statistical analysis of responses of these films to a subset of the target compounds; Caltech provided sensor data for the statistical analysis. The polymers used in the STS-95 flight experiment are listed in Table I.

Protocols for depositing these polymers have been published previously [4], [8]. Because the polymer films are sensitive to changes in temperature [9], heaters were included on the back of the ceramic substrates to provide a constant temperature at the sensors. The substrates were usually heated to a few degrees above ambient temperature at set points of 28,32 , or $36^{\circ} \mathrm{C}$. Thermistor selection prohibited heating above $36{ }^{\circ} \mathrm{C}$. Heating the substrates helped remove some of the thermal drift seen in the sensors and also assisted in vapor desorption and, therefore, sensor recovery time.

\section{Device Design and Construction}

The device to be used in the JPL ENose flight experiment was defined as one capable of monitoring the shuttle cabin environment for the presence of ten contaminants at or below the 1-h SMAC levels. The target compounds are listed in Table II. In addition to the target compounds, the ENose was also built to be capable of detecting changes in humidity.

These compounds were selected by reviewing results of analysis of the constituents of air collected in the space shuttle after a flight [3], and selecting those that had been present in relatively high concentrations, although SMACs for those compounds had not been approached. Additional compounds, such as ammonia, were selected if there had been anecdotal reports from shuttle crewmembers that an odor had been present at some time during a flight. The reported concentrations of these compounds and the 1-h SMACs are listed in Table II.

After the flight experiment was defined, one target compound was added to the list; a medical wipe saturated with $70 \%$ 2-propanol was used to provide a daily event marker, to verify device operation during the flight. The exact composition of the wipe is not known.

The ENose fabricated was a complete, autonomous device, consisting of 32 sensors in an array, electronics to allow control of the array as well as to read the response and transfer data from the array to a computer, and a computer for instrument control, data acquisition and data storage. Design of data analysis software to allow identification and quantification of the target com- pounds list was included in the development program. The original intent of the program was to develop real-time data analysis and display. However, after consultation with the flight experiment co-investigator, Dr. J. James of the Toxicology Branch at Johnson Space Center, it was decided that post-flight analysis would adequately validate the JPL ENose performance, and would be preferable to running the risk of false positive analyses presented to crewmembers. Telemetry for ground analysis during the flight was not available, and data were analyzed after the flight.

\section{Operation}

When the device is operating, air is pumped from the surroundings into the sensor chamber at $0.25 \mathrm{~L} / \mathrm{min}$ using a Thomas model X-400 miniature diaphragm pump. The air is directed either through an activated charcoal filter which is put in line to provide clean air for baseline data, or though a dummy-filter of Teflon beads which is put in line to provide a pressure drop similar to that in the charcoal filter. A solenoid valve can be programmed to open the path to the charcoal filter and provide clean airflow for a programmable period of time at programmable time intervals; otherwise, the air is directed through the Teflon beads. Air then enters the glass enclosed sensor head chamber where resistance is measured. For this experiment, the charcoal filter was used for 15 min every $3.5 \mathrm{~h}$. A sketch of the device layout is found in Fig. 2.

The gas-flow temperature is monitored, and if ambient temperatures rise above the substrate temperature (initially set at $28^{\circ} \mathrm{C}$ ), then the substrate heaters are reset to the next highest temperature set point; conversely, if the ambient temperature dropped below the lower set point, the heaters would be reset to the lower temperature. The heaters would not heat above $36{ }^{\circ} \mathrm{C}$, but it was not expected that the shuttle cabin temperature would rise that high.

Data acquisition and device control are accomplished using a PIC 16C74A microcontroller and a Hewlett Packard HP 200 LX palm top computer, which is programmed to operate the device, control the heaters and to record sensor resistance. Typical normalized resistance change $\left(\Delta R / R_{0}\right)$ for $10-50$ ppm of contaminant is on the order of $2 \times 10^{-4}$ (200 ppm resistance change), and may be as small as $1 \times 10^{-5}$. The resistance measurement circuitry designed for the ENose has been described previously [4]-[9].

\section{DATA ANALYSIS-IDENTIFICATION AND QUANTIFICATION OF COMPOUNDS}

The data analysis focused on development of a method that could correctly identify and accurately quantify a gas event of single or mixed gases. For the JPL ENose system, the task of identifying and quantifying a gas event is roughly a two-step procedure: response pattern extraction and pattern recognition. Response pattern extraction for a gas event identifies a resistance response for each sensor and, subsequently, generates the response pattern of the array to the gas event. Pattern recognition uses the generated response pattern to identify and quantify the gas event. In order to implement these data analysis steps, a database of expected gases must be compiled; this database 
TABLE II

TARGET COMPOUNDS FOR THE JPL ENOSE SHUTTLE EXPERIMENT

\begin{tabular}{l|c|c|c|c}
\hline Compound & $\begin{array}{l}\text { Detected on } \\
\text { shuttle (ppm)[3] }\end{array}$ & $\begin{array}{l}\text { 1 hour SMAC } \\
(\mathbf{p p m})[\mathbf{1 , 2}]\end{array}$ & $\begin{array}{l}\text { Training range } \\
\mathbf{( p p m )}\end{array}$ & $\begin{array}{l}\text { Minimum } \\
\text { Concentration } \\
\text { Detected (ppm) }\end{array}$ \\
\hline methanol & $<1$ & 30 & $5-300$ & 5 \\
\hline ethanol & $0.5-5$ & 2000 & $10-130$ & 50 \\
\hline 2-propanol & $0.4-4$ & 400 & $30-160$ & 50 \\
\hline ammonia & 0 & 30 & $10-50$ & 20 \\
\hline benzene & $<0.1$ & 10 & $10-150$ & 10 \\
\hline indole & 0 & 1 & $.006-.06$ & 0.03 \\
\hline methane & $1.0-10$ & 5300 & $1000-7000$ & 3000 \\
\hline formaldehyde & 0 & 0.4 & $1-510$ & 25 \\
\hline Freon 113 & $0.1-1$ & 50 & $10-600$ & 20 \\
\hline toluene & $0.4-4$ & 16 & $5-60$ & 15 \\
\hline
\end{tabular}

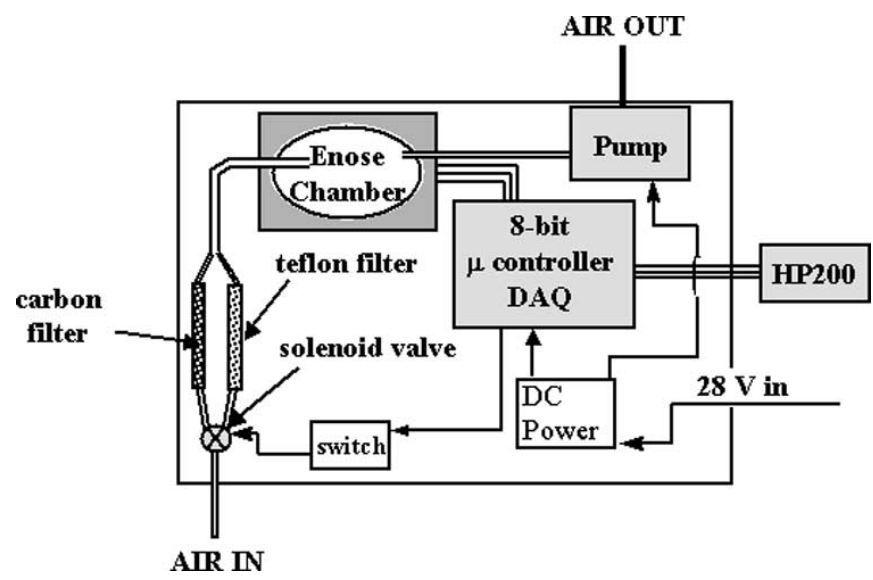

Fig. 2. Schematic of ENose flight experiment device.

will be referred to as our ENose array training data. Algorithms used for pattern recognition and the pattern extraction processes have been discussed else where in detail [6], [17], but will be reviewed here briefly.

\section{A. Data Analysis Algorithm}

Some of the conceptual ENose data analysis approaches tested were as follows: discriminant function analysis (DFA), neural networks with back propagation (NNBP), and a linear algebra (LA) based approach. Principal component analysis was initially used, but was later replaced by DFA (Fisher discriminant) due to its better ability at discriminating similar signatures that contain subtle, but possibly crucial, gas-discriminatory information for our case. Both DFA and NNBP are among the popular methods for array-based sensor data analysis. In general, NNBP is more suitable than DFA when the sensor signatures of two gases are not separable by a hyperplane (e.g., one gas has a signature surrounding the signature of another gas), while DFA is better in classifying data sets which may overlap.

Linear algebra is suitable only if the sensor responses are linear, which was later not to be the case for some sensors for the concentration ranges considered. Though the nonlinearities appear to be of low order and for few single analytes [17], successful identification and quantification of gas events must take the nonlinearities into account. Linearizing the response patterns with the LA-based approach did not improve the identification and quantification much. For this reason, an algorithm based on the Levenberg-Marquart nonlinear least squares (LM-NLS) method was developed [7].

NLS has been studied previously for analysis ion concentrations with an electrode sensor array [18]. However, computation time and convergence problems have prevented it from being used widely for ENose/tongue data analysis. In our case, where we need to deconvolute gas compounds and their concentrations for over 20 potential target gases and mixtures of up to four of them (it would require an enormous amount of training data for a NN based approach) with large variation in response patterns, we found the NLS based approach to be a very effective way. With the fast advance in computer technology, computation time is no longer a big issue. Currently, it takes several minutes $(<5 \mathrm{~min})$ for analysis each event on a nondedicated Pentium III PC with MATLAB 5.3, quick enough to be used for "quasi real time" air monitoring, especially when considering the fact that the ENose sensor's response time is about $15 \mathrm{~min}$ (for 1-h SMAC levels of concentrations of most target gas compounds). This can be further reduced when optimized for real time code ( $<1 \mathrm{~min})$. The convergence problem can be improved by using multiple starting points as well as a modified update strategy and is also relaxed by the fact that the quantification accuracy requirement in our case is rather tolerant $(+/-50 \%$ of the true concentration).

For simplicity, we continue to assume sensors' additive response, i.e., the nonlinearity is assumed to be limited to individual compounds but not their mixtures, as no clear evidence so far show otherwise.

Similar to LA, the LM-NLS method tries to find the best-fit parameter vector $\mathbf{x}$ from an observation vector $\mathbf{y}$, which is related to $\mathbf{x}$ through a known linear or nonlinear function, $\mathbf{y}=\mathrm{f}(\mathbf{A}, \mathbf{x})$. However, unlike LA, where parameter estimation can be obtained analytically, the LM-NLS method needs to use iterative optimization procedures to compute the parameter estimates. It begins from a given starting point of $\mathbf{x}$, calculates the discrepancy of the fit

$$
\text { residual }=\frac{(\text { computed }- \text { observed })}{\sigma}
$$


where $\boldsymbol{\sigma}$ is the standard deviation and updates with a better-fitted parameter $\mathbf{x}$ at each step for a smaller residual. The LM-NLS method automatically adjusts the parameter step to assure a reduction in the residual: increase damping (reduce step) for a highly nonlinear problem, and decrease damping (increase step) for a linear problem. Because of this ability to adjust damping, the LM-NLS method is adaptive to both linear and nonlinear problems. This is a very desirable feature since only limited sensors exhibit nonlinearity in response to some target gases. Details of how this method adjusts damping can be found in [16].

To obtain sensor characteristics without further knowledge of sensor nonlinear mechanisms, a second order polynomial fit was used to model the low-order nonlinearities found. Thus, for each sensor's response to each gas compound, the program finds the best-fit coefficients $\mathbf{A}_{\mathbf{1}}$ and $\mathbf{A}_{\mathbf{2}}$ (in the least-squares sense) to the following equation: resistance change $=\mathbf{A}_{1} \mathbf{x}+\mathbf{A}_{\mathbf{2}} \mathbf{x}^{\mathbf{2}}$, where $\mathbf{x}$ is the gas concentration vector. The fit is constrained to pass through the origin. $\mathbf{A}_{\mathbf{1}}$ and $\mathbf{A}_{\mathbf{2}}$ are $12 \times 32$ matrices characterizing the 32 sensors' response to the ten targeted gases plus humidity change and wipe.

\section{B. Response Pattern Extraction}

In order to extract the resistance response pattern accurately from raw time-series resistance data, it must be preprocessed. This conversion is important because relative response changes have been found to be more reliable than the response shapes for sensing media such as the conducting polymer/carbon films used in this program. The exact method of extracting the response pattern may be application dependent, but, in general, it will involve four sequential steps: 1) noise removal, 2) baseline drifting accommodation, 3) gas event occurrence determination, and 4) resistance change calculation.

1) Noise Removal: A sensor's response to a gas event might be buried in noise, especially at the gas concentrations targeted in this program (1-100 ppm). The main source of this noise is response fluctuation in the sensing films. Some polymer films were noisier than others; that noise could have been caused by high sensitivity of the film to small changes in pressure caused by airflow. Nonuniformities in the film thickness and carbon dispersion could also be responsible for noise. In general, this fluctuation is fast compared to the response to a gas event. It is expected that a less responsive sensing medium will have a larger relative fluctuation. The first step in the preprocessing is therefore to filter out this high frequency fluctuation using appropriate digital filtering.

The method for noise removal used here is zero-phase forward and reverse digital filtering. The length of the filter may be different for different sensors and can be determined by trial and error.

2) Baseline Drift Accommodation: Baseline drift is one of the most difficult problems to be solved in analyzing resistance versus time data from the ENose. The causes for the baseline drift can be multiple, and include variations in temperature, humidity, or pressure, aging of the sensors, and sensor saturation. However, there is at present no clear understanding of the underlying mechanism of any one of the causes, which makes drift-compensating attempts very difficult.

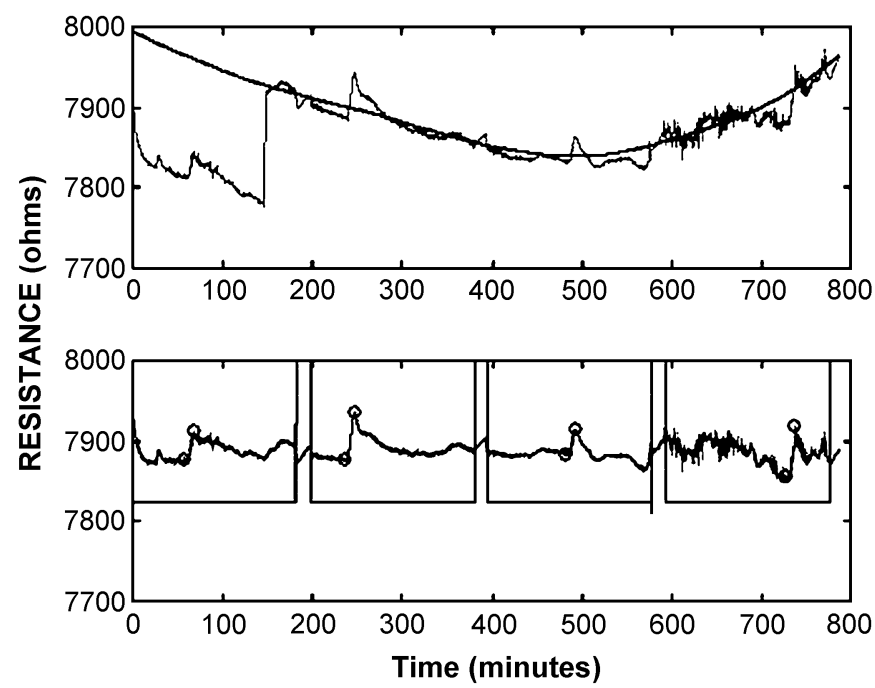

Fig. 3. (Top) Raw resistance data with the baseline drift shown as a smooth curve. (Bottom) Processed resistance data with gas event on/off times indicated by circles.

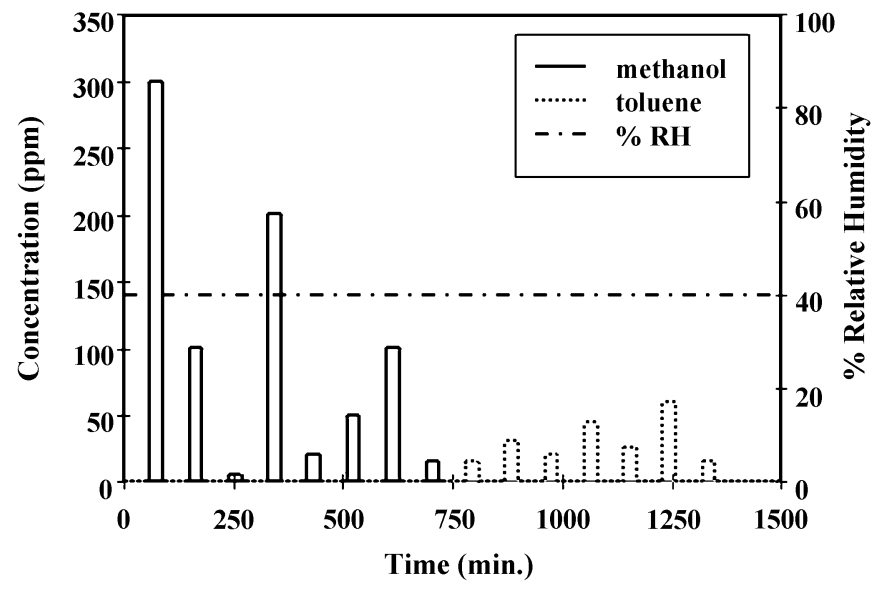

Fig. 4. Typical gas exposure event sequence for training sets. Up to three gases can be delivered simultaneously, and relative humidity can be varied. Training events were typically $30 \mathrm{~min}$ of compound with $60 \mathrm{~min}$ of clean air between them.

In general, the baseline drift is slowly varying in nature compared to the response time of a detectable gas event, whether the drift is caused by temperature, pressure or humidity variations, or some other cause. This difference in time scale enables the use of a long-length digital filter to determine the approximate baseline drift and then subtract it from the raw data.

3) Gas Event Occurrence Determination: A scheme for automated determination of whether and when a gas event occurs has been developed. It is based primarily on threshold calculation, in which the resistance change over a certain time interval is calculated, and a time stamp is registered if the change exceeds a preset threshold. This routine can detect most gas events; however, it was also found that it tends to falsely identify baseline drift or noise as gas events. Consequently, in this experiment, the determination of a gas event in practice was largely done by visual inspection of the events selected by the routine. In this way, effort was focused on gas identification and quantification. 


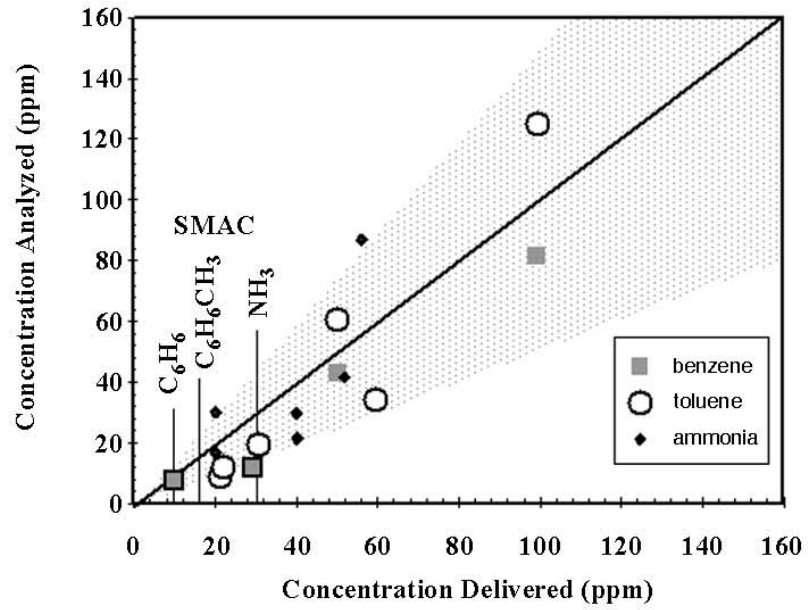

(a)

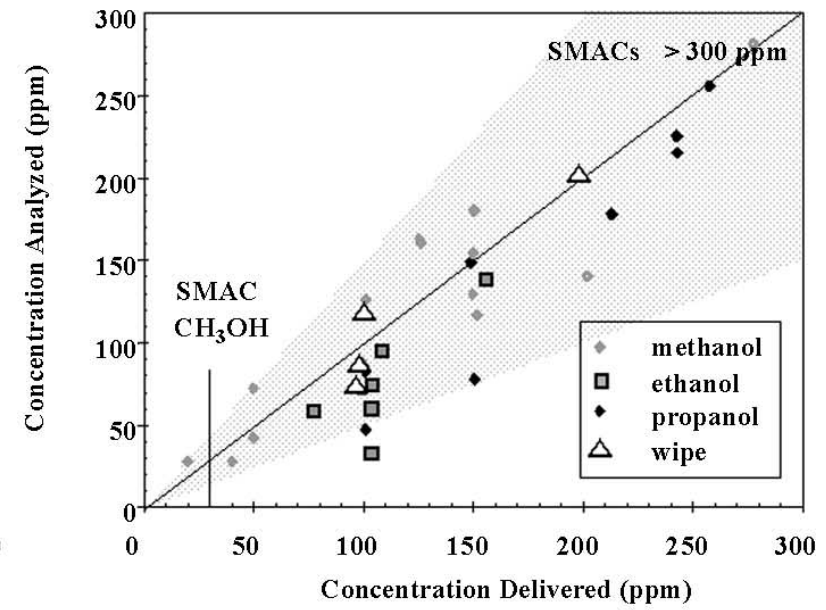

(b)

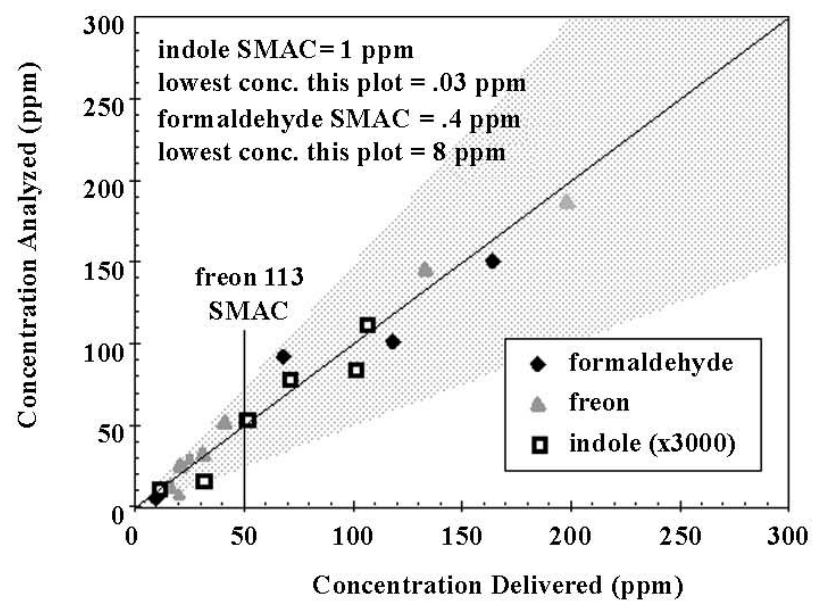

(c)

Fig. 5. For (a), (b), and (c), plots show the delivered concentration versus the concentration quantified by the algorithm. Points within the shaded region are within $50 \%$ of the delivered concentration. (a) Benzene, toluene, and ammonia. (b) Methanol, ethanol, propanol, daily marker, or wipe. (c) Formaldehyde, freon, and indole.

4) Resistance Change Calculation: The calculation of the resistance change may not be obvious, because at low gas concentration range the responses do not have consistent characteristic shapes. However, it is expected that different sensors have relatively different response strengths. It is this relative responsiveness which determines the fingerprint of that gas, i.e., the response pattern. To preserve this relative responsiveness it is important that the any calculation method of the resistance change should be taken at the same time stamp after the initial presentation of a gas.

Fig. 3(a) shows the baseline drift determined by piecewise baseline fitting and low-frequency filtering. Fig. 3(b) shows the result of processing data with high and low frequency filters and correcting for baseline drift. The step at around $150 \mathrm{~min}$ is caused by a change in array temperature and the solid, regularly spaced line show when the carbon filter was selected for developing baseline information. The circles indicate selected event start and finish times.

\section{Training Data}

Data analysis algorithms for identification and quantification of gas events were developed from sets of training data. The training data were taken on two sets of polymer-film sensorarrays. The two arrays were operated in parallel so they would be exposed to the same atmosphere. Both sets were exposed to some 550 events where single gases at varying concentrations in filtered, dehumidified house air were delivered, and 100 mixed gas events.

The data from the training sets were used to construct data analysis algorithms; additional sets were used to test the algorithms after training. The sets were used to construct a database of sensor array responses to extract a signature, or fingerprint, pattern for each gas. The sensors were exposed repeatedly to different concentrations of the target compounds. A typical single gas event sequence exposes the sensor array to a series of concentrations of two gases delivered singly, as shown in Fig. 4. Concentration changes were not made in any set order. The concentration ranges to which the sensor arrays were exposed was approximately $50 \%-300 \%$ of the 1-h SMAC of each gas, although for compounds with high SMACs, such as ethanol, significantly lower concentration ranges were used for compatibility with the laboratory gas control system. The range of training exposure concentrations for each gas is shown in Table II. The bulk of these exposures were done at $0 \% \mathrm{RH}$ at 
$25^{\circ} \mathrm{C}$. In addition to the compounds in Table II, the array was trained to water from $0-90 \% \mathrm{RH}$ at $25^{\circ} \mathrm{C}$. For those compounds with high SMACs, the sensors were also exposed to the saturated vapor pressure of those compounds to determine whether the sensors would saturate. It was not found that any sensors in the array saturated or failed to recover rapidly (typically, $5 \mathrm{~min}$ was sufficient for recovery) from exposure to high concentrations of organic vapors.

An exhaustive set of mixed gas training sets was not attempted, although most of the compounds were trained at additional (besides 0\% RH) humidity backgrounds. Sets of mixtures of two analytes at varying concentrations were recorded to determine whether additivity of response would hold at these concentrations and to determine the capability of the algorithms in deconvoluting the signals.

Before analysis of the analyte exposures, high and low frequency noise are removed by filtering. High frequency noise is largely caused by the responses of the sensing films. Low frequency noise appears in the data as baseline drift, and is largely caused by humidity, temperature and pressure changes in the monitored atmosphere. Baseline drift, which is not removed by high or low frequency filters, is removed by constructing a piece-wise baseline from the signals taken during the baseline determination cycle (cleaned, filtered air) and subtracting it from the data.

Sensor data are recorded as resistance versus time and the events (exposure to an analyte) are analyzed as normalized changes in resistance $\left(\Delta R / R_{0}\right)$ where $R_{o}$ is determined for each gas event for several data points before the event begins. The program calculates the $\Delta \mathrm{R} / \mathrm{Ro}$ values from the processed data. This value is more accurately described as follows:

$$
\frac{\Delta \mathrm{R}}{\mathrm{R}_{\mathrm{o}}}=\frac{\left(\mathrm{R}_{\mathrm{t}}-\mathrm{R}_{\mathrm{o}}\right)}{\mathrm{R}_{\mathrm{o}}}
$$

where

$\mathrm{R}_{\mathrm{t}} \quad$ sensor resistance at plateau of the response;

$R_{0} \quad$ resistance prior to the event.

At the concentration ranges determined from the SMACs, response of the array is not linear with concentration, thus there is no single signature or fingerprint for one gas at all concentrations. The response patterns for one gas remain similar for the concentration range of interest, but are not similar enough to rely on linear analysis methods. In order to account for nonlinear responses, event identification and quantification was done using the LM-NLS method [16]. The data analysis routines and the rationale for selecting this method are discussed in detail elsewhere [6], [17].

\section{RESULTS \& DISCUSSION}

\section{A. Training and Testing the Sensor Array}

The goal of the device development program, and a condition for flight, was the ability of the ENose to identify and quantify the target gases at or below the 1-h SMAC with an $80 \%$ success rate under both dry and humid conditions (30-80\% RH at $25 \mathrm{C})$. "Success" is defined as correct identification of the compound and quantification of the compound within $50 \%$ of the

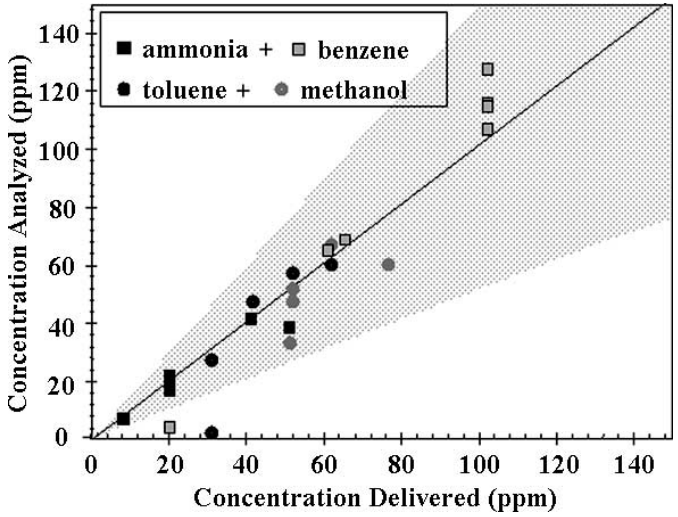

Fig. 6. Results for analyzing two gas mixtures: ammonia + benzene and toluene + methanol. The shaded region indicates $50 \%$ of the delivered gas concentration.

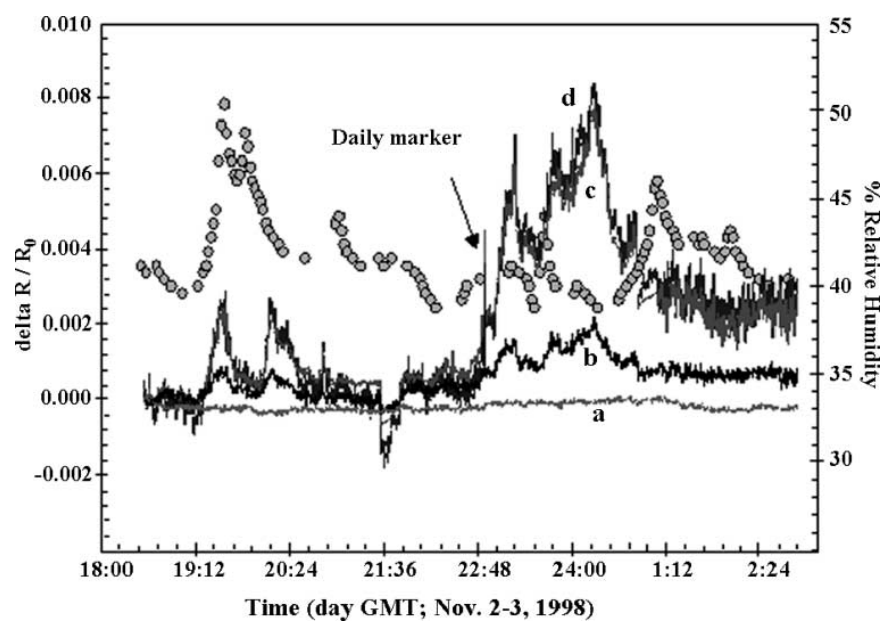

Fig. 7. STS-95 ENose data. Circles: relative humidity. Polymer responses: (a) poly (2, 4, 6-tribromostyrene), (b) polyamide resin, (c) poly (ethylene oxide), and (d) poly (4-vinylphenol). Dip at 306.9 corresponds to 15 -min baseline cycle.

actual concentration. This degree of accuracy, 50\%, is sufficient for crew health applications where the SMACs are set conservatively and toxicity levels are not known with better accuracy.

Two identical sensor arrays were fabricated; one array was arbitrarily designated as the flight array and the second set the spare array. The quantification and identification algorithms were built exclusively from the training data on the flight array, although both arrays were exposed to all the training sets. These algorithms were used to test identification and quantification for both sets of chips.

Fig. 5(a)-(c) show the results of application of the data analysis algorithms to the training data for the flight array. The training data is treated as a blind test and the algorithm identifies and quantifies the data; for the correctly identified gas events, Fig. 5 plots the delivered concentration versus the concentration quantified by the algorithm. The figures show that the data analysis program was able to correctly identify the compound and quantify the compounds in the training set within $+/-50 \%$ of the delivered concentration. The data analysis reports both identity and quantity; the overall success rate in identification and quantification was $85 \%$.

Fig. 6 shows the results of analyzing mixtures of two gases. The success rate with mixed gases was moderate, about $65 \%$. 

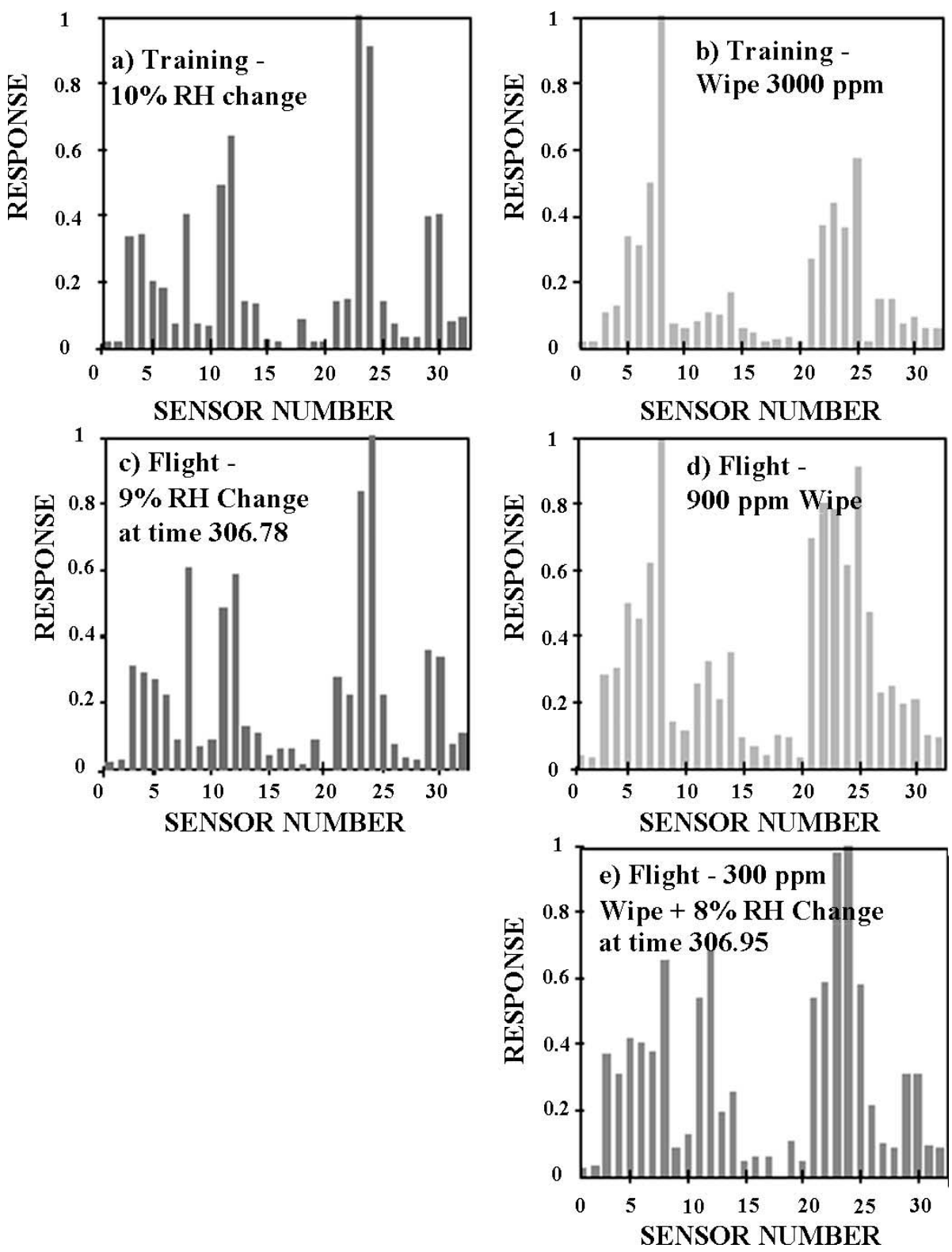

Fig. 8. Fingerprints for flight events compared with training fingerprints.

An exhaustive set of double gas mixtures was not run; additive linearity holds for the mixtures tested: $\{$ ammonia + benzene $\},\{$ ammonia + ethanol $\}$, \{benzene + methanol $\}$, \{methanol + toluene $\}$, $\{$ ethanol + formaldehyde $\}$, and $\{$ propanol + benzene\}.

After the shuttle flight, the sensor chips were stored under ambient laboratory conditions inside the device. The laboratory in which the device was stored saw humidity fluctuations from $20 \%-80 \% \mathrm{RH}$ and from $13-30{ }^{\circ} \mathrm{C}$. One year after the sensors were made, the flight array was retested with all of the target compounds under dry conditions with separate testing for humidity; the algorithms from the original training still correctly identified and quantified all of the compounds. Subsequent testing was done at three-month intervals on a subset of compounds (benzene, toluene, 2-propanol, and relative humidity) to test the lifetime of the sensor array using the original algorithms. At eighteen months, the signature of methanol remains reasonably similar; however, the signatures of benzene and toluene changed enough that the algorithms could no longer correctly identify them. At 22 months, visual inspection of the fingerprints could not detect any change; however, the algorithm could no longer correctly identify the subset of compounds. Lifetime calibrations on the individual sensors were not analyzed.

\section{B. Shuttle-Flight Experiment of the JPL ENose}

The ENose flight experiment monitored the air in the mid-deck (crew quarters) of the orbiter. Monitoring was continuous, three points per minute, and the device required no crew intervention after it was removed from the locker, placed, and turned on with a single switch. The ENose response was recorded over six days and data were stored for post-flight analysis. During a ground experiment done in 1997, it was found that the recycled air was very clean (air analyzed from the shuttle contains contaminants at less than the SMAC level) 
and there were very few events [3], [8], [19], so a daily marker event was added. The medical swab daily marker event was included in the experiment to confirm that the device was working.

The initial, visual examination of the raw flight data showed that the ENose responded to all planned daily marker events with a rapid rise and fall in resistance. Software event identification and data analysis further confirmed all daily marker events. In addition to the planned events, a large number of other events were recorded. Software analysis identified all events, which were not markers, as humidity changes. Fingerprint patterns from the events were compared visually to each other and to laboratory fingerprint patterns for confirmation of the identification.

Most of the ENose measured humidity changes can be correlated in time with humidity changes measured by an independent humidity sensor in the shuttle orbiter. Those events identified as humidity changes, but not correlated with cabin humidity change, are likely to be caused by local humidity changes; that is, changes in humidity near the ENose which were not sufficient to cause a measurable change in cabin humidity. The independent humidity monitor was located in the stairway between the mid-deck and the flight deck, and so would not record any humidity changes localized around the ENose; in addition, there could be a time lag between the two sensors' response because of their different locations. Fig. 7 is a plot of change in resistance versus time of four sensors and cabin humidity. The sensor responses are shown without frequency filtering or baseline correction. By inspection, it can be seen that the humidity changes and ENose response correlate in several places within the data.

After the raw data are processed and analyzed, the humidity event at time 306.8 in Fig. 7 is identified as a 9\% humidity change. The ENose response shows a rise in humidity followed by a drop in humidity at 306.815 , followed by another rise at 306.835. The humidity monitor response rises and drops similarly, although there is a time lag for the humidity change to reach the ENose. The pattern is similar to the $10 \%$ humidity training data. A similar correlation between ENose sensor response and humidity monitor response is seen in the event at 306.97. Humidity rise at 307.05 was localized to the humidity monitor and did not reach the ENose.

Fig. 8 shows the similarities between patterns for events in Fig. 7, and compares them with patterns recorded in training sets for the daily marker and for humidity change. Response patterns for events in the flight data can be seen to be similar for training data and for flight data. Response is normalized to the largest sensor response in each event to keep patterns on a similar scale. The dip in the ENose sensor response at 306.9 is caused by the carbon filter for baseline measurements.

A medical swab marker event quantified at $900 \mathrm{ppm}$ is similar to, but not the same as, a training pattern at $3000 \mathrm{ppm}$; this difference is one of the nonlinear features that must be accommodated in the analysis software. The marker event at time 306.95 in Fig. 7 is analyzed as a combination of humidity and medical swab, and the pattern can be seen to be a combination of the two training patterns.

Software analysis of the flight data did not identify any other target compounds, as single gases or as mixtures. Independent analysis of daily air samples done in the Toxicology lab at JSC confirmed that no target compounds were found in concentrations above the ENose detection threshold.

The correlation between the ground training and in-flight response patterns for both the alcohol wipe and humidity change shows that the operation of the ENose is microgravity insensitive, and thus can be used in a space-based application without further accounting for microgravity effects.

\section{CONCLUSION}

The miniaturized ENose designed and built at JPL has the capacity to detect, identify, and quantify relative humidity changes as well as a suite of ten contaminants at 1-h SMAC levels. The sensing arrays were comprised of polymer-carbon composite sensing films. Using nonlinear analysis techniques, gas events were identified and quantified with an overall success rate of $85 \%$ for single events and $65 \%$ for mixed gas events. Some three-component gas-event combinations were also deconvoluted for identification and quantification. For the current array of polymers, it was possible to train an array, build an algorithm for both identification and quantification, and get a useful lifetime of the sensing array of eighteen months.

The space shuttle air is very clean, which is desirable for the crew, but does not significantly challenge either the response characteristics of the ENose sensor array or the software analysis routines developed for the ENose. It is not surprising that the only changes the ENose saw on STS-95 were humidity changes, and it is because events were not expected that the experiment included the relatively uncontrolled daily marker (alcohol wipe) events. The ENose experiment is judged a success on several counts. The daily marker was identified and quantified. The humidity events were identified and quantified. The crew reported no events that would be expected to induce a response in the ENose. Finally, analysis of the GSCs did not detect any compounds that the ENose should have identified.

For the two compounds the ENose identified on the shuttle flight, water (measured in relative humidity changes) and 2-propanol, the response of the ENose in microgravity was not significantly different from the response seen in laboratory training (before and after the shuttle flight). This result leads us to conclude that the device is microgravity insensitive.

Further work with the JPL ENose will focus on improving the sensor array, further miniaturization of the device, and adaptation of the software for real-time (within $30 \mathrm{~min}$ of an incident) analysis. The goal is to build the JPL ENose to be used as a gas-incident monitor which can be operated with a minimum of crew time and which can be integrated into environmental control systems.

\section{ACKNOWLEDGMENT}

The authors thank Commander C. L. Brown for his execution of the shuttle portion of the experiment: taking grab samples, administering the alcohol wipe marker, and monitoring the ENose. The authors also thank Dr. J. T. James, Johnson Space Center, for his participation in the flight experiment, and Dr. D. Karmon, JPL, for his participation in the development program. 


\section{REFERENCES}

[1] Toxicology Group, Spacecraft Maximum Allowable Concentrations for Airborne Contaminants, Houston, TX, June 1999.

[2] Spacecraft Maximum Allowable Concentrations for Selected Airborne Contaminants. Washington, DC: National Academy, 1994-2000, vol. $1-4$.

[3] J. T. James, T. F. Limero, H. J. Leano, J. F. Boyd, and P. A. Covington, "Volatile organic contaminants found in the habitable environment of the space-shuttle-Sts-26 to Sts-55," Aviation Space Environ. Med., vol. 65, pp. 851-857, Sept. 1994.

[4] M. A. Ryan, M. L. Homer, M. G. Buehler, K. S. Manatt, B. Lau, D. Karmon, and S. Jackson, "Monitoring space shuttle air for selected contaminants using an electronic nose," in 28th Int. Conf. Environmental Systems, Danvers, MA, 1998.

[5] M. A. Ryan, M. G. Buehler, M. L. Homer, K. S. Manatt, B. Lau, S. Jackson, and H. Zhou, "Results from the space shuttle STS-95 electronic nose experiment," in Micro/Nano Technology Conf., Pasadena, CA, 1999.

[6] M. A. Ryan, "Electronic Nose: New Technology Infusion Advanced Environmental Monitoring and Control for Space Station," Jet Propulsion Lab., Pasadena, CA, Apr. 1999.

[7] M. A. Ryan, M. L. Homer, H. Zhou, K. S. Manatt, V. S. Ryan, and S. P. Jackson, "Operation of an electronic nose aboard the space shuttle and directions for research for a second generation device," in Proc. 30th Int. Conf. Environmental Systems, Toulouse, France, 2000.

[8] M. A. Ryan, M. L. Homer, M. G. Buehler, K. S. Manatt, F. Zee, and J. Graf, "Monitoring the air quality in a closed chamber using an electronic nose," in 27th Int. Conf. Environmental Systems, Lake Tahoe, NV, 1997.

[9] M. G. Buehler and M. A. Ryan, "Temperature and humidity dependence of a polymer-based gas sensor," in Proc. Electro-Optical Technology Remote Chemical Detection Identification II, Orlando, FL, July 1997, pp. 40-48.

[10] B. Lundberg and B. Sundqvist, "Resistivity of a composite conducting polymer as a function of temperature, pressure, and environment-applications as a pressure and gas concentration transducer," J. Appl. Phys., vol. 60, pp. 1074-1079, Aug. 1, 1986

[11] K. A. Hu, D. Moffatt, J. Runt, A. Safari, and R. Newnham, "V2O3-polymer composite thermistors," J. Amer. Ceram. Soc., vol. 70, pp. 583-585, Aug. 1987.

[12] G. G. Neuburger and P. C. Warren, "Chemically actuated electronic switch," Sens. Actuators B, vol. 1, pp. 326-332, Jan. 1990.

[13] M. S. Freund and N. S. Lewis, "A chemically diverse conducting polymer-based electronic nose," in Proc. National Academy Sciences United States Amer., vol. 92, Mar. 28, 1995, pp. 2652-2656.

[14] M. C. Lonergan, E. J. Severin, B. J. Doleman, S. A. Beaber, R. H. Grubb, and N. S. Lewis, "Array-based vapor sensing using chemically sensitive, carbon black-polymer resistors," Chem. Mater, vol. 8, pp. 2298-2312, Sept. 1996.

[15] A. Marquez, J. Uribe, and R. Cruz, "Conductivity variation induced by solvent swelling of an elastomer-carbon black-graphite composite," $J$. Appl. Polymer Sci., vol. 66, pp. 2221-2232, Dec. 19, 1997.

[16] M. Lampton, "Damping-undamping strategies for the Levenberg-Marquardt nonlinear least-squares method," Comput. Phys., vol. 11, pp. $110-115$.

[17] H. Zhou, M. A. Ryan, and M. L. Homer, "Nonlinear least squares based algorithm for identifying and quantifying single and mixed air contaminants with JPL's electronic nose," IEEE Sensors J..

[18] C. Di Natale, A. Macagnano, F. Davide, A. D'Amico, A. Legin, Y. Vlasov, A. Rudnitskaya, and B. Selezenev, "Multicomponent analysis on polluted waters by means of an electronic tongue," Sens. Actuators $B$, vol. 44, pp. 423-428, 1997.

[19] P. O. Wieland, "Designing for human presence in space: an introduction to environmental control and life support systems," NASA Ref. Pub., p. $1324,1994$.

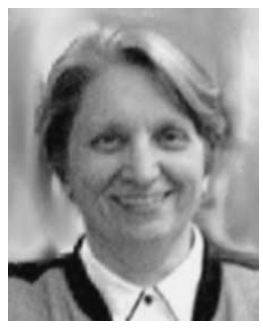

Margaret Amy Ryan received the Ph.D. degree in physical chemistry from the University of Massachusetts, Amherst.

She is the Principal Investigator of the ENose project at the Jet Propulsion Laboratory, Pasadena, CA. Her research interests include chemical sensors, including polymer-based sensors, colorimetric sensors, and silicon carbide-based hydrocarbon sensors, and materials and processes for thermal-to-electric energy conversion, including high-temperature solid electrolytes and metallic and semiconducting

materials.

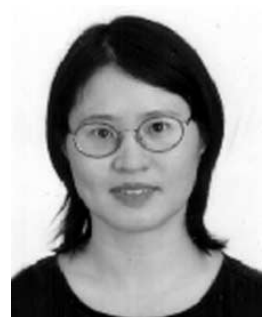

Hanying Zhou received the Ph.D. degree in electrical engineering from The Pennsylvania State University, University Park, in 1995.

She has been with Jet Propulsion Laboratory, Pasadena, CA, since 1998. She has authored and coauthored two book chapters and ten journal papers and numerous conference presentations/papers in the fields of optical information processing and ENose data analysis. Her main research interests are in the fields of optical information processing, including optical correlator, holographic data storage, and electrooptic Fourier transform spectrometers. In addition, she is also active in the field of ENose data analysis for array-based sensors.

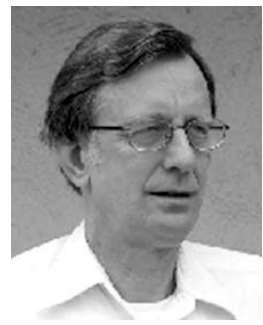

Martin G. Buehler received the B.S.E.E. and M.S.E.E. degrees from Duke University, Durham, $\mathrm{NC}$, in 1961 and 1963, respectively, and the Ph.D. degree in electronic engineering from Stanford University, Stanford, CA, in 1966, specializing in solid-state electronics.

He was with Texas Instruments, Dallas, TX, for six years, the National Bureau of Standards, Boulder, CO, for eight years, and, since 1981, he has been at the Jet Propulsion Laboratory (JPL), Pasadena, CA. At JPL, he is a Senior Research Scientist in the Microdevices Laboratory. Over the past ten years, he has developed more than one instrument per year, including radiation monitors, ENoses, electrometers, and e-tongues. Currently, at JPL, he is serving as a Technologist for the New Millennium Program.

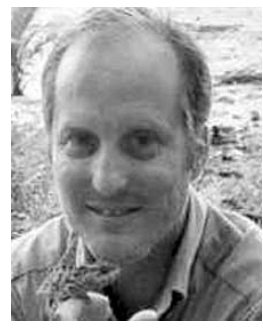

Kenneth S. Manatt received the B.S. degree in geology from the University of California, Santa Cruz.

He has been with the Jet Propulsion Laboratory, Pasadena, CA, since 1987, working on sensing system design and development. He is currently with ACRO services, Pasadena.

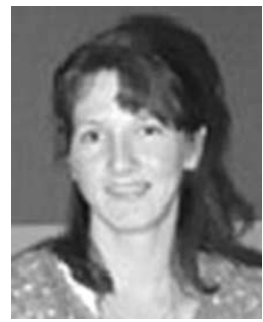

Victoria S. Mowrey received the B.S. degree in marine biology from California State University, Long Beach, in 1997 and the M.S. degree in environmental engineering from the University of Southern California, Los Angeles, in 2000.

She is currently with the Launch Approval Planning Group at the Jet Propulsion Laboratory (JPL), Pasadena, CA, developing environmental and safety documentation for JPL flight projects.

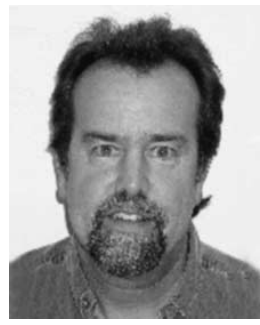

Shannon P. Jackson received the B.S. degree in electrical engineering from the California Polytechnic University, Pomona.

He has been with the Jet Propulsion Laboratory (JPL), Pasadena, CA, since 1985. He holds two patents in the area of signal processing and has been instrumental in developing space flight hardware that has flown on Earth orbit and Mars missions, as well as developing and fielding neutrino detectors in Antarctica. His interests include micropower applications, fiber optics, and wireless systems.

Mr. Jackson has received several National Space Act Awards, the most recent for his work on Sensor Webs. 


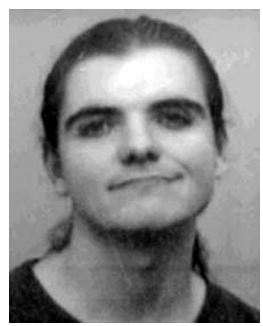

Adam K. Kisor received the B.A. degree in cognitive science and visual arts from the University of California, San Diego.

Since he joined the Jet Propulsion Laboratory, Pasadena, CA, in 1992, he has worked primarily on the alkali metal thermal-to-electric converter (AMTEC) and chemical sensor technology. He is currently an Associate Engineer on the ENose team in which he helps to design, fabricate, and test most of the components.

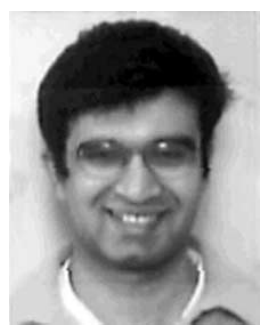

Abhijit V. Shevade received the B.Chem.Engg. and M.Chem.Engg. degrees from the Department of Chemical Technology, University of Bombay, Bombay, India, in 1993 and 1996, respectively, and the Ph.D. degree in chemical engineering from Kansas State University, Manhattan, in 2001.

His research interests include molecular modeling of interfacial phenomena, and he is currently developing a polymer-carbon composite models for the ENose program at the Jet Propulsion Laboratory, Pasadena, CA.

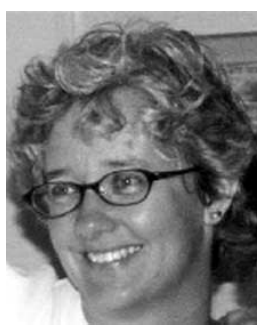

Margie L. Homer received the B.A. degree in chemistry from Swarthmore College, Swarthmore, PA, in 1985 and the Ph.D. degree in physical chemistry from the University of California, Los Angeles, in 1993.

She is the Co-Investigator of the ENose project at the Jet Propulsion Laboratory, Pasadena, CA. Her research interests include chemical sensors. 